\title{
Zur Androgenrezeptor-Hypothese der amyotrophen Lateralsklerose
}

\author{
A. Danek, G. D. Borasio, Th. N. Witt, W. Kreß* \\ Neurologische Klinik, Ludwig-Maximilians-Universität München (Direktor: Prof. Dr. Th. Brandt) \\ * Institut für Humangenetik, Universität Würzburg (Direktor: Prof. Dr. H. Höhn)
}

\section{The Bulbospinal Neuronopathy \\ Androgen Receptor Gene Defect is Not \\ Shared by Patients with Amyotrophic Lateral Sclerosis}

Based on the hypothesis of androgen receptor dysfunction in amyotrophic lateral sclerosis (ALS), the CAG-repeat stretch of exon 1 of the androgen receptor gene was analysed in 12 ALS patients, since abnormal length of this repeat is pathogenic for the motoneuron disease of X-linked bulbospinal neuronopathy. Repeat length in all ALS patients was normal. Therefore, a different defect of motoneuron androgen receptors than abnormal CAG-repeat expansion, if present at all, should be responsible for ALS.

\section{Zusammenfassung}

Unter Annahme einer möglichen Funktionsstörung des Androgenrezeptors bei der amyotrophen Lateralsklerose (ALS) wurde die repetitive CAGTrinukleotidsequenz seines ersten Exons bei 12 Patienten untersucht, da eine abnorme Verlängerung dieses Genabschnitts zur ALS-ähnlichen bulbospinalen Neuronopathie führt. Nach den Ergebnissen kommt dieser Typ vom Androgenrezeptordefekt in der Pathogenese der ALS nicht in Frage: alle Patienten zeigten eine Normalbefund.

\section{Einleitung}

Neben anderen Hypothesen für die ungeklärte Pathogenese der amyotrophen Lateralsklerose (ALS) wird diskutiert, ob eine gestörte Funktion des Androgenrezeptors als pathophysiologische Grundlage vorliegt. Diese Überlegung basiert auf der Entdeckung, daß Motoneurone Testosteron und seine Homologen binden (10), und wird durch das Überwiegen der Erkrankung bei Männern und die Beobachtung, daß sie bei Frauen meist erst nach der Menopause auftritt, epidemiologisch unterstützt (13).

Die X-chromosomal vererbte bulbospinale Neuronopathie (BSN) ist eine seltene, auch als KennedySyndrom bekannte Multisystemerkrankung, die bei Männern beobachtet wird. Klinisch führend ist eine Motoneuronerkrankung mit bulbärer Prädilektion, was gelegentlich zur Fehldiagnose als ALS führen kann (5). Autopsiebefunde zeigen bei BSN ebenso wie bei ALS, daß die Motoneurone der Augenbewegungsnerven, die wenig Androgenrezeptoren aufweisen, verschont werden (11). Die Fertilität bei BSN ist in unterschiedlichem Maße beeinträchtigt (1). Bei einigen Patienten mit BSN können Funktionsstörungen des Rezeptors in Androgenbindungsstudien unter Verwendung skrotaler Fibroblasten nachge- wiesen werden (12). Kürzlich gelang es, eine abnorm verlängerte repetitive Trinukleotidsequenzfolge im ersten Exon des Gens für den Androgenrezeptor als molekulare Grundlage der Erkrankung nachzuweisen (2-4,6-8).

Nachdem die genannten Beobachtungen dem Androgenrezeptor sowohl bei der BSN als auch der ALS eine pathogenetische Rolle zuschreiben, liegt die Vermutung nahe, daß bei beiden Erkrankungen ein vergleichbarer Defekt vorhanden sein könnte. Zur Überprüfung dieser Hypothese untersuchten wir das Gen des Androgenrezeptors bei Patienten mit ALS, um die Länge des repetitiven Sequenzabschnittes zu bestimmen, der bei der BSN abnorm vergrößert und gegenüber Kontrollpersonen annähernd verdoppelt ist.

\section{Methoden}

Lymphozyten-DNS wurde von 12 Patienten (8 Männer, 4 Frauen, 29-77 Jahre alt) mit nach den El-Escorial-Kriterien der World Federation of Neurology klinisch eindeutiger (Befall des ersten und zweiten Motoneurons mit Progredienz) sporadischer ALS gewonnen. Die repetitive CAG-Sequenz (Wiederholungen des Basentriplets Zytosin-Adenin-Guanin) im ersten Exon des Androgenrezeptorgens wurde mit Hilfe der Polymerasekettenreaktion amplifiziert; die Reaktionsbedingungen entsprachen denen bei LaSpada et al. (8). Die Reaktionsprodukte wurden auf einem 3\%-Agarose-Gel aufgetrennt und mit Ethidiumbromid gefärbt. 


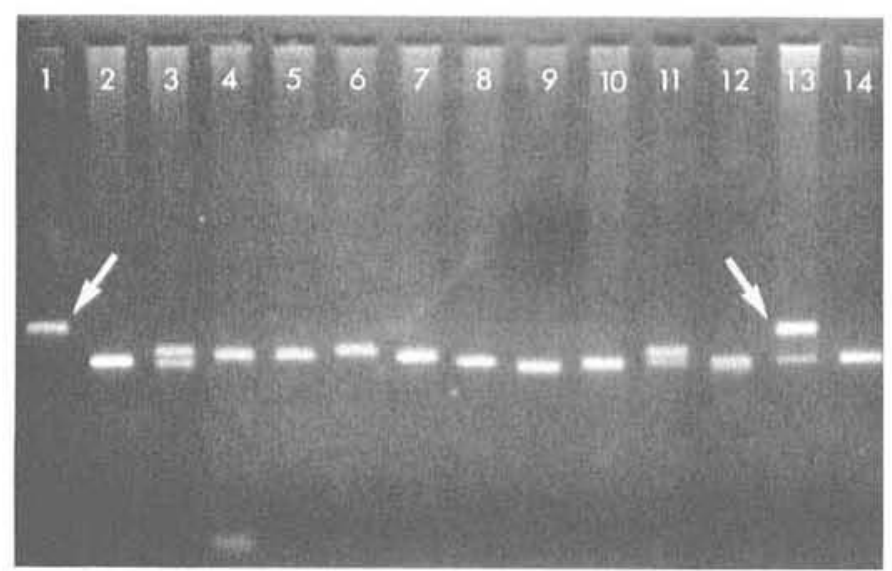

Abb. 1 Der CAG-Block in Exon 1 des X-chromosomalen Androgenrezeptorgens wurde bei 12 Patienten mit ALS (Spuren $2-12,14), 1$ Patienten mit BSN (1) und 1 weiblichen BSN-Überträgerin amplifiziert (13). Durch die verlangsamte Wanderung der Ethidiumbromidgefärbten PCR-Produkte (Pfeile) ist die abnorme Expansion des CAG-Blocks bei BSN erkennbar (elektrophoretische Wanderungsrichtung von oben nach unten). Bei der weiblichen Überträgerin (mit einem betroffenen und einem unbetroffenen X-Chromosom) erkennt man zusätzlich zu diesem verlängerten DNS-Fragment das kleine, normale Allel. Unsere 12 ALS-Patienten zeigten nur normale Allele des Gens. Bei 2 erkrankten Frauen ( 3 und 11 ) hatten die beiden Allele des Gens zwar gering unterschiedliche, aber dennoch normale Längen.

\section{Ergebnisse und Diskussion}

Bei keinem Patienten mit ALS fand sich eine abnorme Expansion der CAG-Sequenz, wie sie für BSN typisch ist. Abb. 1 zeigt die Ergebnisse der 12 ALSPatienten im Vergleich mit den Befunden eines Patienten mit BSN und einer weiblichen Überträgerin dieser Erkrankung.

Zusammenfassend schließen wir, daß die ALS einen anderen zur Motoneuronerkrankung führenden pathophysiologischen Mechanismus als die BSN aufweist. Da hier nur ein begrenzter Bereich des ersten Exons untersucht wurde, ist aber eine Funktionsstörung der Androgenrezeptoren auf Motoneuronen von ALS-Patienten nicht generell ausgeschlossen. Ergänzend ist darauf hinzuweisen, daß bei familiärer, autosomal-dominant vererbter ALS kürzlich Mutationen im Gen einer Superoxiddismutase als molekulares Korrelat wahrscheinlich gemacht werden konnten (9).

\section{Literatur}

${ }^{1}$ Arbizu, T., J. Santamaria, J. M. Gomez et al.: A family with adult spinal and bulbar muscular atrophy, X-linked inheritance and associated testicular failure. J. Neurol. Sci. 59 (1983) $371-382$

${ }^{2}$ Biancalana, V., F. Serville, J. Pommier et al.: Moderate instability of the trinucleotide repeat in spino bulbar muscular atrophy. Hum. Molec. Genet. 1 (1992) 255-258

${ }^{3}$ Doyu, M., G. Sobue, E. Mukai et al.: Severity of X-linked recessive bulbospinal neuronopathy correlates with size of the tandem CAG repeat in androgen receptor gene. Ann. Neurol. 32 (1992) 707-710

${ }^{4}$ Grüner, B. M., C. R. Müller: Genkartierung und molekulare Diagnose der X-chromosomal rezessiven bulbospinalen Muskelatrophie (Typ Kennedy). Akt. Neurol. 19 (1992) 70-72

5 Harding, A. E., P. K. Thomas, M. Baraitser et al.: X-linked recessive bulbospinal neuronopathy: a report of ten cases. $J$. Neurol. Neurosurg. Psychiatry 45 (1982) 1012-1019

6 Igarashi, S., Y. Tanno, O. Onodera et al.: Strong correlation between the number of CAG repeats in androgen receptor genes and the clinical onset of features of spinal and bulbar muscular atrophy. Neurology 42 (1992) 2300-2302

T LaSpada, A. R., D. B. Roling. A. E. Harding et al.: Meiotic stability and genotype-phenotype correlation of the trinucleotide repeat in X-linked spinal and bulbar muscular atrophy. Nature Genet. 2 (1992) 301-304

${ }^{8}$ LaSpada. A. R., E. M. Wilson, D. B. Lubahn et al.: Androgen receptor gene mutations in X-linked spinal and bulbar muscular atrophy. Nature 352 (1991) 77-79

${ }^{9}$ Rosen, D. R., T. Siddique, D. Patterson et al.: Mutations on $\mathrm{Cu} /$ $\mathrm{Zn}$ superoxide dismutase gene are associated with familial amyotrophic lateral sclerosis. Nature 362 (1993) 59-62

10 Sar, M., W. E. Stumpf: Androgen concentration in motor neurons of cranial nerves and spinal cord. Science 197 (1977) $77-80$

11 Sobue, G., Y. Matsuoka, E. Mukai et al.: Spinal and cranial motor nerve roots in amyotrophic lateral sclerosis and X-linked recessive bulbospinal muscular atrophy: Morphometric and teased-fiber study. Acta Neuropathol. (Berlin) 55 (1981) $227-235$

12 Warner, C. L., J. E. Griffin, J. D. Wilson et al.: X-linked spinomuscular atrophy: A kindred with associated abnormal androgen receptor binding. Neurology 42 (1992) 2181-2184

13 Weiner. L. P.: Possible role of androgen receptors in amyotrophic lateral sclerosis, a hypothesis. Arch. Neurol. 37 (1980) 129-131 